\title{
Telepsiquiatria como metodologia alternativa no internato médico no interior do Tocantins: um relato de experiência
}

Telepsychiatrics as an alternative methodology in the medicine internship in the interior of Tocantins: an experience report

\section{La telepsiquiatría como metodología alternativa en la pasantía médica en el interior de Tocantins: un informe de experiencia}

Thiago Henrique Silva ${ }^{1 *}$, Ricardo Rodrigues Goulart ${ }^{1}$, Guilherme Fernandes Ribeiro ${ }^{1}$, Rodrigo Santalucia Bonjardim², Carlos Alberto Rangearo Peres ${ }^{1}$.

\section{RESUMO}

Objetivo: llustrar uma vivência obtida por estudantes de graduação em medicina com a telepsiquiatria e o método de ensino à distância como parte das atividades do internato médico durante a pandemia da COVID19. Relato de experiência: São relatadas as atividades realizadas por 74 discentes em diversos municípios interioranos do estado do Tocantins/Brasil dentro do módulo de internato rural. Os estudantes foram capazes de atender pacientes e intermediar o contato entre a equipe de psiquiatria oferecida pela universidade e enfermos que por vezes vivem em longínquos municípios empregando como ferramenta a telepsiquiatria, além da realização das discussões clínicas e devolutivas ao paciente supervisionado por preceptor através do método de ensino à distância. No decorrer do internato, os acadêmicos identificaram e selecionaram pacientes, realizaram anamneses prévias e acompanharam os pacientes psiquiátricos durante a teleconsulta nos seus respectivos municípios, sendo ofertado à população várias consultas gratuitas com profissionais especialistas. Considerações finais: Essa metodologia permitiu aos pacientes acesso a um acompanhamento psiquiátrico especializado que alguns dificilmente teriam em seu município. Também proporcionou que os estudantes acompanhassem as consultas e a evolução do paciente ao longo do tempo, contribuindo para seu aprendizado e formação profissional, substituindo a metodologia tradicional impossibilitada pela COVID-19.

Palavras-chave: Telepsiquiatria, Educação à distância, Internato médico.

\begin{abstract}
Objective: To illustrate an experience obtained by medical students with telepsychiatry and the distance learning education as part of the activities of the medical internship during the COVID-19 pandemic. Experience report: The activities carried out by 74 medical students in several country municipalities in the state of Tocantins/Brazil within the medical internship are reported. The students were able to assist patients and mediate the contact between the psychiatric team offered by the university with the patient who often lives in a distant city, using telepsychiatry as a tool, in addition to conducting clinical discussions and feedback to the patient supervised by a preceptor through the distance learning education. During the internship the students identified and selected patients, performed previous anamnesis and accompanied psychiatric patients during teleconsultation in their respective municipalities, offering to the population several free consultations with specialists. Final considerations: This method allowed patients to have access to specialized psychiatric follow-up that some would hardly have in their county. Additionally, allowed the students to follow the patient's treatment and evolution over time, contributing to their learning and professional training, replacing the traditional methodology made impossible by COVID-19.
\end{abstract}

Keywords: Telepsychiatry, Distance learning education, Medical internship.

1 Universidade Federal do Tocantins (UFT), Palmas - TO. *E-mail: thiagohensi@gmail.com

2 Universidade Federal da Grande Dourados (UFGD), Dourados - MS. 


\section{RESUMEN}

Objetivo: llustrar una experiencia obtenida por estudiantes de medicina con la telepsiquiatría y la educación a distancia como parte de las actividades de la pasantía médica durante la pandemia de COVID-19. Informe de experiencia: Se reportan las actividades realizadas por 74 estudiantes de medicina en varios municipios rurales del estado de Tocantins/Brasil dentro de la pasantía médica. Los estudiantes pudieron asistir a los pacientes y mediar el contacto entre el equipo de psiquiatría ofrecido por la universidad con el paciente que vive en una ciudad lejana, utilizando la telepsiquiatría como herramienta, además de realizar discusiones clínicas y retroalimentación al paciente supervisado por un preceptor a través de la educación a distancia. Durante la pasantía los estudiantes identificaron y seleccionaron pacientes, realizaron anamnesis previa y acompañaron a pacientes psiquiátricos durante la teleconsulta en sus respectivos municipios, ofreciendo a la población varias consultas gratuitas con especialistas. Consideraciones finales: Este método permitió a los pacientes tener acceso a un seguimiento especializado que algunos difícilmente tendrían en su condado. Además, permitió al alumno seguir la conducta y evolución del paciente a lo largo del tiempo, contribuyendo a su aprendizaje y formación profesional, reemplazando la metodología tradicional imposibilitada por el COVID-19.

Palabras clave: Telepsiquiatría, Educación a distancia, Pasantía médica.

\section{INTRODUÇÃO}

Em fevereiro de 2020, o Ministério da Saúde confirmou no Brasil o primeiro caso da doença causada pelo novo coronavírus, conhecida pela sigla COVID-19, uma patologia de alto potencial de contágio e patogenicidade, demandando isolamento social da população e quarentena dos infectados (BRASIL, 2020).

A necessidade de medidas restritivas visando frear o contágio exigiu da sociedade mudança em seu modo de vida e obrigou as instituições de ensino superior (IES) a adaptarem seus planos pedagógicos (FERREL MN e RYAN JJ, 2020; GONDIM MPMC, et al., 2021). Foi necessário que universidades, departamentos acadêmicos e cursos universitários se adequassem para reduzir o dano pedagógico e riscos à saúde pública, enquanto paradoxalmente garantiam a manutenção de uma educação em nível superior de qualidade e com segurança (GUSSO HL, et al., 2020).

Dentre as adaptações necessárias, muitas IES foram obrigadas a paralisar suas atividades presenciais (FUZARI WMP, et al., 2021; GUSSO HL, et al., 2020). O United Nations International Children's Emergency Fund citado por Gondim MPMC, et al. (2021) relatou que a educação de 1 bilhão e 570 milhões de estudantes em mais de 190 países foi interrompida, correspondendo a $91 \%$ de todos os estudantes no mundo. As atividades presenciais suspensas em universidades públicas e privadas implicam a necessidade de desenvolvimento de maneiras alternativas de ensino, destacando-se a implementação de sistemas digitais (GUSSO HL, et al., 2020).

A educação médica também precisou adaptar-se (CHINELATTO LA, et al., 2020). Em março de 2020 a American Association of Medical Colleges (AAMC) posicionou-se recomendando que estudantes de graduação em medicina não deveriam participar em nenhum atendimento direto ao paciente (AAMC, 2020). Vários modelos foram utilizados para suspensão das aulas, como o de algumas faculdades de medicina que interromperam completamente suas atividades, do ensino básico ao internato, enquanto outras mantiveram somente os alunos do sexto ano com atividades práticas e os demais migraram para diversos modelos de educação a distância (EAD), geralmente com uso da internet (CHINELATTO LA, et al., 2020).

Alterações abruptas e indefinições quanto ao modelo de ensino a ser implementado contribuíram com a ansiedade e estresse em muitos estudantes, que foram duplamente penalizados pela pandemia, mudando seu modo de vida em sociedade e pelas restrições impostas para contenção da COVID-19 e convivendo com incertezas sobre o futuro de sua formação médica (SHAHRVINI B, et al., 2021). O debate acerca da estruturação do currículo médico focado em um paradigma pouco articulado com as revoluções das tecnologias da informação e comunicações já ocorria havia algum tempo, porém, a necessidade de migração para o EAD impulsionado pela pandemia tornou a questão mais contundente (BIRCH E e DE WOLF M, 2020). 
Apesar de o ensino remoto ter possibilitado a continuidade dos cursos de medicina, seu uso ainda é contraditório entre estudantes e professores. Em uma pesquisa realizada com 2721 estudantes no Reino Unido concluiu que para os estudantes os maiores benefícios do EAD são a otimização de tempo, a flexibilidade e a possibilidade de que cada aluno aprenda em seu ritmo, enquanto apontou a distratibilidade do ambiente residencial e a má qualidade das conexões com a internet como principais barreiras (DOST S, et al., 2020).

Os últimos dois anos da formação médica no Brasil são compostos pelo internato, período em que o acadêmico de graduação terá maior contato com pacientes e treinará habilidades necessárias à sua prática profissional, e enquanto plataformas online são mais adequadas para o início da graduação médica, ressaltase a importância do contato com o paciente para os estudantes do internato, uma vez que diversas habilidades do fazer clínico não são conseguidas na modalidade virtual, sendo fundamental a interação direta com o paciente na formação do raciocínio clínico (MIAN A e KHAN S, 2020; MORETTI-PIRES RO, et al., 2021).

A telemedicina surge como vertente da ciência médica cada vez mais necessária, principalmente em vista de circunstâncias excepcionais como a da pandemia da COVID-19, pois oferece uma alternativa para a continuidade do atendimento médico, com uma forma heterodoxa de contato médico-paciente sem risco de disseminação de doenças de contato direto (LOEB AE, et al., 2020; TANAKA MJ, et al., 2020).

Como citado por Weber AM, et al. (2021) a inserção do estudante de graduação em medicina nesse contexto pode ajudar a suprir parcialmente o déficit das atividades práticas, e Parisien RL, et al. (2020) acrescenta que paralelamente permite ao médico continuar o cuidado dos pacientes. $O$ uso da telemedicina conjugada a psiquiatria reduz os efeitos da falta de profissionais especializados, limitações geográficas e da falta de expertise de médicos da atenção básica que atendem pacientes com afecções psiquiátricas (O'KEEFE M, et al., 2021).

Este relato tem como objetivo relatar e expor a vivência de estudantes da graduação em medicina em um contexto misto de telepsiquiatria e EAD durante a pandemia da COVID-19. Tal experiência ocorreu no módulo de internato rural (MIR) de universidade pública da região central do Tocantins, em cidades interioranas deste estado, durante os meses de setembro a dezembro de 2020.

\section{RELATO DE EXPERIÊNCIA}

O projeto pedagógico do curso de medicina da universidade prevê que os últimos quatro semestres letivos sejam realizados em regime de internato, priorizando atividades práticas e contato com os pacientes nos diversos níveis da atenção, bem como em diversos modelos de atendimento médico. Para tal, se estrutura em sete módulos com duração de treze semanas sendo o MIR o último a ser realizado, subdividindo-se em três atividades: quatro semanas de acompanhamento e práticas em hospital universitário próprio de médio porte na região norte do estado; quatro semanas de atividades em psiquiatria realizados na cidade sede da faculdade, e; quatro semanas de acompanhamento na saúde em diversas cidades de menor porte do interior do estado, individualmente ou em pequenos grupos.

Após ter suas atividades suspensas em março de 2020 devido à COVID-19, com a mudança das conjunturas envolvidas, os órgãos deliberativos da universidade decidiram em setembro daquele ano que era momento de retomar as atividades do internato. Visando evitar aglomerações e contágios de estudantes e pacientes pelo vírus e avaliando uma melhor situação epidemiológica no interior do Tocantins, foi optado por manter apenas uma turma realizando internato na cidade sede da instituição, a que estava mais adiantada, enquanto outras duas turmas, totalizando 74 alunos, realizariam o MIR no interior do estado. Devido ao forte impacto da pandemia no município do hospital universitário, foi preferido não receber alunos neste nosocômio como tradicionalmente fazia. Assim sendo, estes estudantes foram alocados em cidades interioranas, onde deveriam atuar junto às equipes da atenção básica e nos demais serviços de saúde ofertados no município, como Unidades de Pronto Atendimento, hospitais de nível secundário, mutirões e as diversas ações em saúde.

A equipe docente de psiquiatria, para mitigar possíveis prejuízos decorrentes da suspensão total das práticas em saúde mental, antes realizadas na capital estadual, propôs um modelo misto de ensino. Os alunos foram divididos em quatro grupos de 18 a 20 acadêmicos baseados na localização geográfica das cidades, 
as aulas teóricas seriam ministradas por EAD e as práticas seriam realizadas por telepsiquiatria com a presença física de um interno junto ao paciente e remota sincrônica de psiquiatra docente da faculdade ou de residente de psiquiatra de universidade parceira do estado de São Paulo. Os estudantes deveriam selecionar nos municípios do seu estágio pacientes com demandas psiquiátricas, realizar uma anamnese prévia, esclarecer sobre o termo de consentimento livre esclarecido e encontrar estrutura para a realização da consulta (sala que permitisse a privacidade, conexão com a internet e recursos para transmissão sincrônica de imagem e voz).

Em geral, os alunos selecionaram os pacientes entre os registros de encaminhamento para consultas psiquiátricas na unidade de saúde, realizaram abordagem telefônica inicial, agendaram primeiro contato físico no qual explicavam sobre o programa, colhiam dados do paciente, esclareciam sobre o consentimento formal do paciente, colhiam sua assinatura nesse documento e agendavam o teleatendimento. $O$ discente então acompanhava fisicamente o paciente durante a realização da teleconsulta psiquiátrica com o médico, que durava cerca de trinta minutos, variando caso a caso.

Finalizado o atendimento, o acadêmico entrava em sala de reunião virtual junto ao médico que atendeu o paciente, demais estudantes daquele grupo do MIR e psiquiatra docente da UFT, onde era feita a discussão dos casos e formulado o plano de ações, o qual o discente depois encaminhava ao médico do município que elaboraria o melhor plano de cuidado e realizava a devolutiva com o paciente. Boa parte dos sinais físicos manifestados pelo paciente, como tremor em mãos e sudorese, só poderiam ser notados pelo discente que Ihe acompanhava, o que elevava a importância do estudante na discussão. Durante todos os contatos, os acadêmicos eram orientados a respeitar medidas de segurança do contexto da pandemia.

As discussões contemplaram diversos quadros clínicos, sendo possível discussão sobre os casos por todo o grupo. Diagnósticos como transtorno de estresse pós-traumático, transtorno depressivo maior, transtorno bipolar de humor, transtorno por abuso de substâncias, transtorno de pânico, transtorno ansioso generalizado e transtorno obsessivo compulsivo puderam ser vivenciados pelos discentes através de sua experiência e pela de seus colegas. A posterior discussão do caso com o médico no município, em geral preceptor do aluno no MIR, também era rica. Cada estudante teve a oportunidade de apresentar ao menos um caso, geralmente com acompanhamento longitudinal do paciente, possibilitando avaliar resposta deste ao tratamento proposto e adequando o plano de cuidados.

A experiência vivenciada por cada estudante foi enriquecida também pela presença de múltiplas realidades dos pacientes em seus diferentes domicílios. Como exemplo, em cidade do nordeste do estado com aproximadamente 23 mil habitantes segundo dados do IBGE (2021), considerado município médio na realidade do estado e de papel central em sua Rede de Atenção à Saúde (RAS), contava com atendimento psiquiátrico semanal em Centro de Atenção Psicossocial (CAPS). Aproveitou-se a estrutura do CAPS para realização do atendimento e o plano de cuidados foi entregue ao especialista médico que ali atendia. $\mathrm{Na}$ ocasião da devolutiva, o discente estava presente e teve a oportunidade de acompanhar essa consulta e as demais do dia, enriquecendo ainda mais seu aprendizado em psiquiatria.

A cidade supracitada contou com 10 estudantes e em um caso específico, um discente pode acompanhar cinco atendimentos com a mesma paciente, observando e relatando ao grupo as dificuldades iniciais da paciente com o plano de cuidados, como fora a relação com o profissional na cidade, a evolução e o desfecho breve do caso, o que sem dúvidas foi proveitoso para o aprendizado do grupo.

Simultaneamente, em município da região central do Tocantins com cerca de 5 mil habitantes conforme dados do IBGE (2021); houve realidade divergente. Uma mesma unidade de saúde realizava todos os atendimentos médicos, se dividindo entre atenção básica e atendimentos emergenciais. Esta unidade de saúde contava com psicólogo que atuava diariamente, mas os enfermos que demandavam atendimento psiquiátrico especializado eram encaminhados para município distinto. Nesse contexto, o teleatendimento foi o único contato do estudante com uma consulta especializada em psiquiatria, mas ele pode discutir o caso com a psicóloga, obtendo uma visão interdisciplinar. As histórias e opiniões foram compartilhadas nas reuniões com o grupo, ampliando os conhecimentos de todos nas diferentes realidades de cidades interioranas do Tocantins. Relatamos também que o plano de ações era sempre planejado com base nos recursos ao alcance do paciente. 
A opinião geral dos estudantes foi positiva, transparecendo que se tratou de uma forma válida para diminuir o impacto da falta de práticas em psiquiatria no modelo tradicional. Não houve queixas da estrutura para realização dos atendimentos, sempre encontrando apoio dos órgãos municipais e condições satisfatórias para a realização da teleconsulta, sendo relatado algumas falhas na conexão com a internet, não impeditivas aos atendimentos. Também foram frequentes reações positivas pelos profissionais de saúde dos municípios, sendo o plano de ações quase sempre integralmente implementado. Foi relatado satisfação por parte dos pacientes, o que ficou nítido no em seus retornos.

\section{DISCUSSÃO}

O impacto da epidemia na formação médica é preocupação global, com ênfase para desafios que envolvem a não integralização da carga horária preconizada, o deslocamento de práticas para campos diversos e os desafios com plataformas EAD, em um contexto que exige adaptação e simultaneamente respeita os projetos pedagógicos dos cursos e Diretrizes Curriculares Nacionais (DCN) (DE OLIVEIRA SS, et al., 2020). O nosso relato de caso apresenta uma forma de adequação a esse cenário, reduzindo prejuízos na formação médica.

Admite-se ser impossível que todo o objetivo da aprendizagem se efetive em modalidades a distância. Segundo Gusso HL, et al. (2020) um exemplo é "acolher o sofrimento de outra pessoa", que costumeiramente pertence às disciplinas na área da saúde. Dessa forma, a transição para um modelo totalmente remoto implica prejuízo na formação médica, sendo essencial a manutenção de atividades práticas presenciais. Nosso modelo misto apresentou sucesso ao reduzir o contato entre estudantes e professores, algo necessário visto que segundo Hermes FS, et al. (2021) até hoje não existe tratamento profilático com potencial comprovado para a COVID-19 e na ocasião não havia imunização por meio de vacinas. Paralelamente permitiu que os discentes tivessem interações diretas com pacientes e desenvolvessem suas habilidades médicas.

Importante citar que o Conselho Federal de Medicina (CFM) autorizou em caráter de excepcionalidade a teleorientação, telemonitoramento e teleinterconsulta somente no ano de 2020 (CFM, 2020). A telemedicina apresenta como vantagem aos pacientes psiquiátricos a redução do tempo de deslocamento até grandes centros, redução de dias de trabalho perdido, menores custos de deslocamento sem redução da eficácia (ACIERNO R, et al., 2017; MORLAND LA, et al., 2017). Essa modalidade esteve ausente na maioria das faculdades médicas e não foi encontrado outro relato na UFT, portanto nossa experiência também foi útil para que os estudantes tivessem contato com essa ferramenta vanguardista.

A pandemia da COVID-19 pode impactar a saúde mental devido à substancial exposição ao estresse psicossocial, sendo possível prever um aumento na incidência de doenças mentais (BROWN E, et al., 2020). Segundo Nascimento AH, et al. (2021) a maioria das psicopatologias é manejada na atenção básica, local que também concentra os médicos recém-formados, portanto o ensino da psiquiatria se mantém como essencial. Seria de grande prejuízo para os estudantes se um método prático alternativo não tivesse sido disponibilizado.

O MIR tem por vocação auxiliar na saúde dos municípios participantes. Em especial nessa ocasião, a telepsiquiatria ajudou a cobrir a demanda de atendimento especializado, inclusive reduzindo custos e facilitando o acesso de populações carentes e que vivem distante de grandes centros. Importante citar que apesar de amplamente utilizado durante a pandemia, o teleatendimento como recurso em saúde mental precisa ser validado, bem como definir o paciente apto a recebe-lo e criar escalas e instrumentos que melhorem a avaliação não presencial (MINERVINO AJ, et al., 2020).

A pandemia impôs necessidade de modificações no modelo tradicional tanto do atendimento médico quanto do internato. A telemedicina, em especial a telepsiquiatria, vem se mostrando como alternativa viável neste contexto para a manutenção das consultas. Ao expormos os benefícios da telepsiquiatria para estudantes, pacientes e municípios, podemos concluir que nossa experiência com teleatendimento foi uma alternativa inovadora com impacto educacional positivo na área psiquiátrica para futuros clínicos gerais. Adicionalmente, proporcionou contato dos discentes com esta tecnologia. 


\section{AGRADECIMENTOS}

Agradecemos a todo corpo docente de psicologia e psiquiatria da Universidade Federal do Tocantins pelo seu esforço em manter alto nível de educação.

\section{REFERÊNCIAS}

1. ACIERNO R, et al. A non-inferiority trial of Prolonged Exposure for posttraumatic stress disorder: In person versus home-based telehealth. Behaviour Research and Therapy, 2017; 89: 57-65.

2. IBGE. Instituto Brasileiro de Geografia e Estatística. Cidades. Brasília: Aparecida do Rio Negro/Tocantins. 2021. Disponível em: https://cidades.ibge.gov.br/brasil/to/aparecida-do-rio-negro/panorama. Acesso em 30 de maio de 2021.

3. ASSOCIATION OF AMERICAN MEDICAL COLLEGES (AAMC). Guidance on Medical Students' Participation in Direct Patient Contact Activities. Washington: AAMC, 2020. Disponível em: https://www.aamc.org/system/files/202008/meded-August-14-Guidance-on-Medical-Students-on-Clinical-Rotations.pdf\#: :text=2.-

,Medical\%20students\%20participating\%20in\%20direct\%20patient\%20contact\%20activities\%20as\%20part,administra tive\%2Fstaff\%20support\%2C\%20are\%20all. Acesso em 30 de maio de 2021.

4. BIRCH E, DE WOLF M. A novel approach to medical school examinations during the COVID-19 pandemic. Medical Education Online, 2020; 25(1): 1785680.

5. BRASIL. Guia de vigilância epidemiológica Emergência de saúde pública de Importância nacional pela Doença pelo coronavírus 2019 - covid-19. Brasília: Ministério da Saúde, 2021. Disponível em: https://www.conasems.org.br/wpcontent/uploads/2021/03/Guia-de-vigila\%CC\%82ncia-epidemiolo\%CC\%81gica-da-covid_19_15.03_2021.pdf. Acesso em: 30 de maio de 2021.

6. BROWN E, et al. The potential impact of COVID-19 on psychosis: A rapid review of contemporary epidemic and pandemic research. Schizophrenia Research, 2020; 222: 79-87.

7. CHINELATTO LA, et al. What You Gain and What You Lose in COVID-19: Perception of Medical Students on their Education. Clinics, 2020; 75: e2133.

8. CONSELHO FEDERAL DE MEDICINA (CFM). OFÍCIO CFM No 1756/2020 - COJUR. Brasília: CFM, 2020. Disponível em: https://portal.cfm.org.br/images/PDF/2020_oficio_telemedicina.pdf. Acesso em: 30 de maio de 2021.

9. DE OLIVEIRA SS, et al. As Escolas Médicas e os desafios da formação médica diante da epidemia brasileira da COVID-19: das (in)certezas acadêmicas ao compromisso social. APS EM REVISTA, 2020; 2(1): 56-60.

10. DOST S, et al. Perceptions of medical students towards online teaching during the COVID-19 pandemic: a national cross-sectional survey. BMJ Open, 2020; 10: e042378.

11. FERREL MN, RYAN JJ. The Impact of COVID-19 on Medical Education. Cureus, 2020; 12.

12. FUZARI WMP, et al. Atuação do farmacêutico clínico frente à COVID-19 em um hospital público da região amazônica. Revista Eletrônica Acervo Saúde, 2021; 13(5): e6450.

13. GONDIM MPMC, et al. Impact of Coronavirus Disease on the Ophthalmology Residency Training in Brazil. Arquivos Brasileiros de Oftalmologia, 2021; 84(3): 297-298.

14. GUSSO HL, et al. Ensino Superior Em Tempos De Pandemia: Diretrizes À Gestão Universitária. Educação \& Sociedade, 2020; 41: 1-27.

15. HERMES FS, et al. Análise de tratamentos profilático para a COVID-19: uma revisão integrativa. Revista Eletrônica Acervo Saúde, 2021; 13(5): e7167.

16. LOEB AE, et al. Departmental Experience and Lessons Learned With Accelerated Introduction of Telemedicine During the COVID-19 Crisis. The Journal of the American Academy of Orthopaedic Surgeons, 2020; 28(11): e469-e476.

17. MIAN A, KHAN S. Medical education during pandemics: a UK perspective. BMC Medicine, 2020; 18(1): 100.

18. MINERVINO AJ, et al. Desafios em saúde mental durante a pandemia: relato de experiência. Revista Bioética, 2020; 28(4): 647-654.

19. MORETTI-PIRES RO, et al. Estratégias pedagógicas na educação médica ante os desafios da Covid-19: uma revisão de escopo. Revista Brasileira de Educação Médica, 2021; 45(1).

20. MORLAND LA, et al. Telehealth and eHealth interventions for posttraumatic stress disorder. Current Opinion in Psychology, 2017; 14: 102-108.

21. NASCIMENTO AH, et al. Relato de experiência acerca do cuidado integral de portadores de sofrimento psíquico na atenção primária de saúde. Revista Eletrônica Acervo Saúde, 2021; 13(5): e7150.

22. O'KEEFE M, et al. Asynchronous telepsychiatry: A systematic review. Journal of Telemedicine and Telecare, 2021; 27(3): 137-145.

23. PARISIEN RL, et al. Telehealth Utilization in Response to the Novel Coronavirus (COVID-19) Pandemic in Orthopaedic Surgery. The Journal of the American Academy of Orthopaedic Surgeons, 2020; 28(11): e469-e476.

24. SHAHRVINI B, et al. Pre-clinical remote undergraduate medical education during the COVID-19 pandemic: a survey study. BMC Medical Education, 2021; 21(1): 13.

25. TANAKA MJ, et al. Telemedicine in the Era of COVID-19: The Virtual Orthopaedic Examination. The Journal of bone and joint surgery. 2020; 102(12): e57.

26. IBGE. Instituto Brasileiro de Geografia e Estatística. Cidades. Brasília: Tocantinópolis/Tocantins. 2021. Disponível em: https://www.ibge.gov.br/cidades-e-estados/to/tocantinopolis.html. Acesso em 30 de maio de 2021.

27. WEBER AM, et al. An outpatient telehealth elective for displaced clinical learners during the COVID-19 pandemic. BMC Medical Education, 2021;21(1): 174. 